\title{
On the Navier-Stokes problem
}

\author{
Alexander G. Ramm \\ Department of Mathematics, Kansas State University, \\ Manhattan, KS 66506, USA \\ ramm@math.ksu.edu \\ http://www.math.ksu.edu/ ramm
}

\begin{abstract}
One of the millennium problems is discussed. The results of the author's solution to this problem are explained. The problem discussed is the Navier-Stokes problem in the whole space.
\end{abstract}

\section{Introduction}

There is a large literature on the Navier-Stokes (NS) problem in $\mathbb{R}^{3}$ ( see [2], [3] and references therein.) The global existence and uniqueness of a solution in $\mathbb{R}^{3}$ was not proved for a long time. It is mentioned as one of the millennium problems in [3]. This problem was solved in [9]. The goal of this paper is to explain briefly the new ideas in this solution and formulate the results of this solution. Therefore parts of paper [9] are used verbally.

The NS problem in $\mathbb{R}^{3}$ consists of solving the equations

$$
v^{\prime}+(v, \nabla) v=-\nabla p+\nu \Delta v+f, \quad x \in \mathbb{R}^{3}, t \geq 0, \quad \nabla \cdot v=0, \quad v(x, 0)=v_{0}(x) .
$$

Vector-functions $v=v(x, t), f=f(x, t)$ and the scalar function $p=p(x, t)$ decay as $|x| \rightarrow \infty$ uniformly with respect to $t \in \mathbb{R}_{+}:=[0, \infty), v^{\prime}:=v_{t}, \nu=$ const $>0$, the velocity $v$ and the pressure $p$ are unknown, $v_{0}$ and $f$ are known, $\nabla \cdot v_{0}=0$. Equations (1) describe viscous incompressible fluid with density $\rho=1$. By $\bar{v}$ complex conjugate of $v$ is denoted.

We assume that $|f|+|\nabla f|+\left|v_{0}\right|+\left|\nabla v_{0}\right|$ decay sufficiently fast as $|x| \rightarrow \infty, \int_{0}^{\infty} N_{0}(f) d t<\infty$ and $N_{0}(f):=\|f\|_{L^{2}\left(\mathbb{R}^{3}\right)}$. By $\nabla v$ any of the first derivatives of $v$ is understood.

We use the integral equation for $v$ :

$$
v(x, t)=F-\int_{0}^{t} d s \int_{\mathbb{R}^{3}} G(x-y, t-s)(v, \nabla) v d y .
$$

Equation (2) is equivalent to (1), see [7]. Formula for the tensor $G$ is derived in [7]. The term $F=F(x, t)$ depends only on the data $f$ and $v_{0}$ (see equation (18) in [7]):

$$
F:=\int_{\mathbb{R}^{3}} g(x-y) v_{0}(y) d y+\int_{0}^{t} d s \int_{\mathbb{R}^{3}} G(x-y, t-s) f(y, s) d y .
$$

MSC: 35Q30; 76D05.

Key words: Navier-Stokes equations; global existence and uniqueness. 
The new idea in the author's solution to to NS problem is to use the Fourier transformed equation (2) and a new a priori estimate for $v$. We assume throughout that $f$ and $v_{0}$ are such that $F$ is bounded in all of the norms we use.

The author's results in [9] can be described as follows:

a) The solution to NS problem exists for all $t \geq 0$ in a suitable Banach space $X$ of smooth functions and is unique in $X$.

b) The solution depends continuously in a suitable norm on the data. It is assumed that the data are smooth and decaying sufficiently fast as $|x| \rightarrow \infty$, uniformly with respect to $t \in[0, T]$, where $T>0$ is an arbitrary large fixed number.

A consequence of these results is the conclusion that turbulent motion of fluid cannot occur in the whole space, that is, in the space where no bodies with boundaries are placed.

Let $X$ be the Banach space of continuous functions with respect to $t$ with values in $L^{2}\left(\mathbb{R}^{3}\right)$ with the norm $\|v\|=\|v\|_{X}:=\sup _{t \in[0, T]}\|v(x, t)\|_{L^{2}\left(\mathbb{R}^{3}\right)}$, where $T>0$ is an arbitrary large fixed number.

The new a priori estimate of the solution $v$ is

$$
N_{0}^{2}(\nabla v):=\int|\nabla v(x, t)|^{2} d x \leq C, \quad \int:=\int_{\mathbb{R}^{3}},
$$

where $C>0$ is a constant and the estimate (4) holds uniformly with respect to $t \in[0, T]$. If the data decay sufficiently fast with respect to $x$ and $t$ then $C$ does not depend on $t$.

Let us prove that $\psi(t):=N_{0}(\nabla v) \leq c(t)$.

Take the Fourier transform of $(2)$, denote $\tilde{v}(\xi, t):=(2 \pi)^{-\frac{3}{2}} \int_{\mathbb{R}^{3}} e^{-i \xi \cdot x} v(x, t) d x$ and let $\tilde{G}$ denote the Fourier transform of $G$. Then

$$
\tilde{v}=\tilde{F}-\int_{0}^{t} \tilde{G}(\xi, t-s) \tilde{v} \star(-i \xi \tilde{v}) d s:=B(\tilde{v}) .
$$

Here $\star$ denotes the convolution in $\mathbb{R}^{3}$ and for brevity we omitted the tensorial indices: instead of $\tilde{G}_{m p} \tilde{v}_{j} \star\left(-i \xi_{j}\right) \tilde{v}_{p}$, where one sums up over the repeated indices, we wrote $\tilde{G}(\xi, t-s) \tilde{v} \star(-i \xi \tilde{v})$.

Let $\psi(t):=N_{0}(\nabla v)=N_{0}(|\xi| \tilde{v}), N_{0}(v)=N_{0}(\tilde{v})$, where the Parseval's identity is used. From (5) one gets

$$
\psi(t) \leq N_{0}(|\xi| \tilde{F})+c \int_{0}^{t} N_{0}(|\xi||\tilde{G}(\xi, t-s)|) \psi(s) d s .
$$

Here and below $c$ stands for various constants independent of $t$; we have used the a priori estimate $\sup _{t \geq 0} N_{0}(\tilde{v}) \leq c_{0}$ and the standard estimate $|\tilde{v} \star \tilde{w}| \leq N_{0}(\tilde{v}) N_{0}(\tilde{w})$. One can check that

$$
N_{0}(|\tilde{G}(\xi, t-s)|) \leq c[\nu(t-s)]^{-\frac{3}{4}}, \quad N_{0}(|\xi||\tilde{G}(\xi, t-s)|) \leq c[\nu(t-s)]^{-\frac{5}{4}} .
$$

In the derivation of (7) we use the estimate $|\tilde{G}(\xi, t)| \leq c e^{-\nu t \xi^{2}}$, where $|G|$ is a norm of the matrix, see formula (9) in [7]. We use below s ome lemmas proved in [9].

Lemma 1. The operator $A f:=\int_{0}^{t}(t-s)^{p} f(s) d s$ in the Banach space $X_{0}:=C([0, T])$ has spectral radius $r(A)=0$ for $p>0$ and any fixed $T>0,0 \leq t \leq T$.

Lemma 2. Let $A$ be a linear operator in a Banach space $X$. If $f=A f+f_{0}$ and $r(A)=0$, then $f=\sum_{j=0}^{\infty} A^{j} f_{0}$ for any element $f_{0} \in X$. If $f_{0}=0$ then $f=0$.

Denote $\Phi_{\lambda}(t):=\frac{t_{+}^{\lambda-1}}{\Gamma(\lambda)}, \lambda \neq 0,-1,-2, \ldots$, where $t_{+}^{\lambda-1}:=0$ for $t<0$ and $t_{+}^{\lambda-1}:=t^{\lambda-1}$ for $t>0$. This $\Phi_{\lambda}$ is defined as a distribution, $\Phi_{\lambda} \star \Phi_{\mu}=\Phi_{\lambda+\mu}, \Phi_{\lambda} \star \Phi_{-\lambda}=I$, where $\star$ here denotes 
the convolution in $[0, \infty)$ and $I$ is the identity operator whose kernel is the $\delta$-function. The convolution of the distribution $\Phi_{\lambda}(t)$ with any distribution vanishing for $t<0$ is well defined, see [1]. This holds also in the case when the convolution integral diveres in the classical sense.

The following lemma allows one to claim that a solution of an integral equation (inequality) with a strongly singular kernel solves an integral equation (inequality) with an integrable kernel, see $[8]$.

Lemma 3.If $h=h_{0}+\Phi_{-\lambda} \star h$, then $h=\Phi_{\lambda} \star h-\Phi_{\lambda} \star h_{0}, \quad \lambda>0$.

One has $\int_{0}^{t}(t-s)^{-\frac{5}{4}} \psi d s=\Gamma\left(-\frac{1}{4}\right) \Phi_{-\frac{1}{4}} \star \psi$, where the convolution is in $\mathbb{R}_{+}:=[0, \infty)$.

Inequality (6) can be written as

$$
\psi \leq \psi_{0}+c \Gamma\left(-\frac{1}{4}\right) \Phi_{-\frac{1}{4}} \star \psi, \quad \psi_{0}:=N_{0}(|\xi| \tilde{F})
$$

and $\Gamma\left(-\frac{1}{4}\right)=-4 \Gamma(3 / 4):=-b^{-1}, b>0$.

Let us prove (4). Applying to (8) the operator $\Phi_{1 / 4} \star$ and multiplying by $c^{-1} b$ one gets

$$
\psi \leq c^{-1} b \Phi_{1 / 4} \star \psi_{0}-c^{-1} b \Phi_{1 / 4} \star \psi .
$$

Using Lemmas 1 and 2 one derives from (9) by iterations that

$$
\psi(t) \leq h(t)
$$

where $h(t)$ is the unique solution of the equation

$$
h=c^{-1} b \Phi_{1 / 4} \star \psi_{0}-c^{-1} b \Phi_{1 / 4} \star h .
$$

Equation (11) is solvable by iterations and the iterations converge by Lemma 2. The solution $h$ is bounded by a constant depending only on the data, that is, on $f, v_{0}$ and $T, 0 \leq t \leq T$. By (10) the $\psi$ is bounded by $h$. Since $T>0$ is arbitrary, estimate (4) is proved.

Using formulas (2), (5) and (4) one can estimate $|v(x, t)|$ and $|\tilde{v}(\xi, t)|$.

Since problem (1) is equivalent to (2) and to (5), it is sufficient to prove that (5) has a solution and this solution is unique in $X$.

Uniqueness proof. Let $\tilde{v}_{1}$ and $\tilde{v}_{2}$ solve equation (5). Define

$$
w:=\tilde{v}_{1}-\tilde{v}_{2}, \quad N_{0}(w):=q, \quad N_{0}(|\xi| w):=Q .
$$

Subtracting from equation (5) for $\tilde{v}_{1}$ equation (5) for $\tilde{v}_{2}$, one gets the equation

$$
w=-\int_{0}^{t} \tilde{G}\left[w \star\left(-i \xi \tilde{v}_{2}\right)+\tilde{v}_{2} \star(-i \xi w)\right] d s,
$$

where the $\star$ is the convolution in $\mathbb{R}^{3}$. Taking the norm $N_{0}$ of both sides of (13), denoting $z:=q+Q$, using estimates (4) and formulas (7) and (12) one derives the following relation:

$$
z \leq c\left(\Gamma(1 / 4) \Phi_{1 / 4} \star z+\Gamma\left(-\frac{1}{4}\right) \Phi_{-\frac{1}{4}} \star z\right)
$$

where $\star$ here denotes the convolution in $[0, \infty)$. The operator $\Phi_{\lambda} \star$ with $\lambda>0$ maps the set of non-negative functions (or distributions) into itself, it preserves inequality sign. Applying 
the operator $\Phi_{1 / 4} \star$ to $(14)$, taking into account that $\Gamma\left(-\frac{1}{4}\right)=-4 \Gamma(3 / 4), \Phi_{\lambda} \star \Phi_{\mu}=\Phi_{\lambda+\mu}$, and $\Phi_{\lambda} \star \Phi_{-\lambda}=I$, one gets:

$$
4 c \Gamma(3 / 4) z \leq c \Gamma(1 / 4) \Phi_{1 / 2} \star z-\Phi_{1 / 4} \star z .
$$

It follows from (15) and (10) that $z=0$ (because $f_{0}=0$ in our case, see Lemma 2). This proves the uniqueness of the solution to problem (1) in the space $X$ for any $T>0,0 \leq t \leq T$.

Existence proof. Let us show that the operator $B$ in (5) is a contraction in $X$ for a sufficiently small $\tau, 0 \leq t \leq \tau$. With $w=\tilde{v}_{1}-\tilde{v}_{2}$ one obtains from (5) the following equation:

$$
B\left(\tilde{v}_{1}\right)-B\left(\tilde{v}_{2}\right)=-\int_{0}^{t} \tilde{G}\left[w \star\left(-i \xi \tilde{v}_{1}\right)+\tilde{v}_{2} \star(-i \xi w)\right] d s .
$$

Here the $\star$ denotes the convolution in $\mathbb{R}^{3}$. Using the second estimate (4) one derives:

$$
\left|\tilde{v}_{2} \star(-i \xi w)\right| \leq N_{0}(w) N_{0}\left(\left(|\xi|+\left|\xi^{\prime}\right|\right) \tilde{v}_{2}\left(\xi^{\prime}\right)\right) \leq c N_{0}(w)\left(1+|\xi|^{2}\right)^{1 / 2} .
$$

Using estimates (4), (7) and (17), one obtains from (16) the following inequality:

$$
N_{0}\left(B\left(\tilde{v_{1}}\right)-B\left(\tilde{v_{2}}\right)\right) \leq c\left(\int_{0}^{t}(t-s)^{-\frac{3}{4}} q d s+\int_{0}^{t}(t-s)^{-\frac{5}{4}} q d s\right), \quad q=N_{0}(w) .
$$

Using the formula $t^{-\frac{5}{4}}=-4 \Gamma\left(\frac{3}{4}\right) \Phi_{-\frac{1}{4}}$, rewrite (18) as

$$
N_{0}\left(B\left(\tilde{v}_{1}\right)-B\left(\tilde{v}_{2}\right)\right) \leq c\left(\Gamma(1 / 4) \Phi_{1 / 4} \star q-4 \Gamma(3 / 4) \Phi_{-\frac{1}{4}} \star q\right)
$$

where the $\star$ denotes the convolution in $[0, \infty)$. Applying $\lambda_{1} \Phi_{1 / 4} \star$ to $(19)$, one gets

$$
\lambda_{1} \Phi_{\frac{1}{4}} \star N_{0}\left(B\left(\tilde{v_{1}}\right)-B\left(\tilde{v}_{2}\right)\right) \leq \lambda_{1} c \Gamma(1 / 4) \Phi_{1 / 2} \star q-q, \quad \lambda_{1}^{-1}:=4 c \Gamma(3 / 4)>0 .
$$

Since $q \geq 0$ one has

$$
\lambda_{1} \Phi_{\frac{1}{4}} \star N_{0}\left(B\left(\tilde{v_{1}}\right)-B\left(\tilde{v_{2}}\right)\right) \leq \lambda_{1} c \Gamma(1 / 4) \Phi_{1 / 2} \star q .
$$

From (21) one derives

$$
N_{0}\left(B\left(\tilde{v_{1}}\right)-B\left(\tilde{v_{2}}\right)\right) \leq c \Gamma(1 / 4) \Phi_{1 / 4} \star 1 \sup _{t \in[0, \tau]} q(t) .
$$

Clearly, $\lim _{t \rightarrow 0} \Phi_{1 / 4} \star 1=0$. Thus, $B$ is a contraction on a ball $B_{R}, B_{R}:=\{q: q \leq R\}$, if $\tau$ is sufficiently small, $0 \leq t \leq \tau$, and $R>0$ is an arbitrary large fixed number. Consequently, the operator $B$ in (5) is a contraction on $B_{R}$ for $t \leq \tau$. Therefore the solution to (5) exists in $X$ for $t \leq \tau$. Estimate (4) does not depend on $\tau \leq T$. Therefore one can repeat the argument for $\tau \leq t \leq 2 \tau$ considering the initial value to be $\tilde{v}(\xi, \tau)$ and the free term to be $\tilde{F}(\xi, t), t \in[\tau, 2 \tau]$. The solution $\tilde{v}$ is unique in $X$, as we have proved. So, one gets the existence of the solution on $0 \leq t \leq 2 \tau$. Continue this process and in finitely many steps get the existence of the unique in $X$ solution in $[0, T]$. Since $T>0$ is arbitrary, the solution exists for all $T>0$.

It follows from Theorem 2 that there cannot be turbulent motions of fluid in the NS problem in the whole space $\mathbb{R}^{3}$ if the data $f$ and $v_{0}$ are smooth and rapidly decaying. 


\section{References}

[1] I. Gel'fand and G. Shilov, Generalized functions, Vol.1, AMS Chelsea Publ., 1964.

[2] O. A. Ladyzhenskaya, The mathematical theory of viscous incompressible flow, Gordon and Breach, New York, 1969.

[3] P.Lemarie-Rieusset, The Navier-Stokes problem in the 21-st century, CRC Press, Boca Raton, 2016.

[4] A.G.Ramm, Large-time behavior of the weak solution to 3D Navier-Stokes equations, Appl. Math. Lett., 26 (2013), $252-257$

[5] A.G.Ramm, Existence and uniqueness of the global solution to the Navier-Stokes equations, Applied Math. Letters, 49, (2015), 7-11.

[6] A.G.Ramm, Large-time behavior of solutions to evolution equations, Handbook of Applications of Chaos Theory, Chapman and Hall/CRC, 2016, pp. 183-200 (ed. C.Skiadas).

[7] A.G.Ramm, Global existence, uniqueness and estimates of the solution to the Navier-Stokes equations, Applied Math. Letters, 74, (2017), 154-160

[8] A.G.Ramm, Existence of the solutions to convolution equations with distributional kernels, Global Journal of Math. Analysis, 6(1), (2018), 1-2.

[9] A.G.Ramm, Solution of the Navier-Stokes problem, Appl. Math. Lett., 87, (2019), 160-164. 